\title{
Buprenorphine, methadone, and morphine treatment during pregnancy: behavioral effects on the offspring in rats
}

This article was published in the following Dove Press journal:

Neuropsychiatric Disease and Treatment

6 March 2015

Number of times this article has been viewed

\author{
Hwei-Hsien Chen ${ }^{1,2, *}$ \\ Yao-Chang Chiang ${ }^{3,4, *}$ \\ Zung Fan Yuan ${ }^{5,6}$ \\ Chung-Chih Kuo ${ }^{5,6}$ \\ Mei-Dan Lai ${ }^{2}$ \\ Tsai-Wei Hung' \\ Ing-kang $\mathrm{Ho}^{1,3,4}$ \\ Shao-Tsu Chen ${ }^{2,7}$ \\ 'Center for Neuropsychiatric \\ Research, National Health Research \\ Institutes, Miaoli County, Taiwan; \\ ${ }^{2}$ Master and $\mathrm{PhD}$ Program in \\ Pharmacology and Toxicology, Tzu Chi \\ University, Hualien, Taiwan; ${ }^{3}$ Center \\ for Drug Abuse and Addiction, \\ China Medical University Hospital, \\ ${ }^{4} \mathrm{Graduate}$ Institute of Clinical Medical \\ Science, China Medical University, \\ Taichung, Taiwan; ${ }^{5}$ Master Program \\ in Physiological and Anatomical \\ Medicine, ${ }^{6}$ Department of Physiology, \\ School of Medicine, Tzu Chi \\ University, ${ }^{7}$ Department of Psychiatry, \\ Buddhist Tzu Chi General Hospital, \\ Hualien, Taiwan \\ *These authors contributed equally \\ to this work
} to this work

\begin{abstract}
Methadone and buprenorphine are widely used for treating people with opioid dependence, including pregnant women. Prenatal exposure to opioids has devastating effects on the development of human fetuses and may induce long-term physical and neurobehavioral changes during postnatal maturation. This study aimed at comparing the behavioral outcomes of young rats prenatally exposed to buprenorphine, methadone, and morphine. Pregnant Sprague-Dawley rats were administered saline, morphine, methadone, and buprenorphine during embryonic days 3-20. The cognitive function, social interaction, anxiety-like behaviors, and locomotor activity of offsprings were examined by novel object recognition test, social interaction test, light-dark transition test, elevated plus-maze, and open-field test between 6 weeks and 10 weeks of age. Prenatal exposure to methadone and buprenorphine did not affect locomotor activity, but significantly impaired novel object recognition and social interaction in both male and female offsprings in the same manner as morphine. Although prenatal exposure to methadone or buprenorphine increased anxiety-like behaviors in the light-dark transition in both male and female offsprings, the effects were less pronounced as compared to that of morphine. Methadone affected elevated plus-maze in both sex, but buprenorphine only affected the female offsprings. These findings suggest that buprenorphine and methadone maintenance therapy for pregnant women, like morphine, produced detrimental effects on cognitive function and social behaviors, whereas the offsprings of such women might have a lower risk of developing anxiety disorders.
\end{abstract}

Keywords: prenatal, buprenorphine, methadone, cognitive function, social behavior, anxiety

\section{Introduction}

Children born to heroin- or morphine-addicted mothers have a high mortality rate and deficiency in the central nervous system. ${ }^{1,2}$ These children may present with long-term neuropsychological consequences associated with dysfunction in intellectual ability and emotional control during their school years., ${ }^{3,4}$ Methadone (6-(dimethylamino)4,4-diphenylheptan-3-one) is a synthetically derived agonist that binds to the $\mu-, \kappa-$, and $\delta$-opioid receptors, ${ }^{5}$ thereby exerting morphine-like effects. Currently, methadone maintenance treatment is the most commonly used pharmacotherapy for opioid dependence, including in pregnant women. Methadone maintenance treatment in pregnant women results in lower maternal morbidity/mortality rates and promotes fetal stability and growth compared with women who use heroin. ${ }^{6-8}$ However, methadone maintenance treatment during pregnancy is still associated with poorer neonatal outcomes compared to drug-free controls. ${ }^{9}$

Buprenorphine is a semisynthetic thebaine derivative, which selectively binds to the $\mu$-opioid receptor as a partial agonist, to the nociceptin receptor as a full/partial
Correspondence: Shao-Tsu Chen Department of Psychiatry, Buddhist Tzu Chi General Hospital,

707, Section 3, Chung-Yang Road, Hualien 97004, Taiwan

$\mathrm{Tel}+8863856 \quad 825$ ext 2207

Fax +8863857 716।

Email shaotsu.tw@yahoo.com.tw 
agonist, and to the $\kappa$-opioid receptor as an antagonist. ${ }^{10,11}$ High doses (2-32 $\mathrm{mg}$ ) of buprenorphine have also been used to treat opioid dependence in humans. Clinical studies demonstrated that buprenorphine maintenance during pregnancy showed lower risk of neonatal abstinence syndrome, lesser dose of morphine treatment for reducing neonatal abstinence syndrome, and shorter periods of hospitalization of newborns than methadone treatment. ${ }^{12,13}$ However, controversial findings have been reported. Kahila et $\mathrm{al}^{14}$ demonstrated that severe neonatal abstinence syndrome and need for morphine replacement therapy were seen in $57 \%$ of the buprenorphine-exposed newborns and a high number of sudden infant deaths were also seen to occur. These findings may imply that higher doses of buprenorphine may induce complex effects on the development of the offspring and that more extensive investigations are needed.

Additionally, maternal drug use may continue to have an impact on the child's cognitive, educational, emotional, and behavioral development. ${ }^{3,4}$ Methadone or buprenorphine treatment shows beneficial effects for heroin addicts who are pregnant than patients without treatment. ${ }^{9}$ However, the long-term psychiatric consequences of intrauterine exposure to methadone or buprenorphine on offsprings remain inconclusive. Methadone maintenance in pregnant women did not affect the intellectual and cognitive functioning at 4 years of age, ${ }^{15,16}$ but resulted in heightened activity or energy level, impulsivity, brief attention span, and persistence in preschool children, ${ }^{17}$ and poorer achievement, increased aggression, and disruptions at school. ${ }^{18}$

There are a myriad of confounding medical, pharmacological, genetic, and environmental variables within the children who are prenatally exposed to methadone or buprenorphine. Therefore, it is warranted to compare the behavioral manifestations associated with prenatal exposure to methadone or buprenorphine in animals. Previous studies have shown that prenatal exposure to methadone and buprenorphine induced the tolerance of antinociceptive effects to morphine in the tail-flick test, ${ }^{19,20}$ whereas methamphetamine-induced behavioral sensitization was only significantly increased in prenatally buprenorphine-exposed offspring at their adulthood. ${ }^{21}$ It remains unclear whether offsprings prenatally exposed to these two therapeutic opioids are prone to develop cognitive dysfunction and mood disorders in the same manner.

The opioid receptors have been implicated in learning, ${ }^{22-24}$ social behaviors, ${ }^{25,26}$ and mood-related disorders, ${ }^{22,27,28}$ as well as pain and addiction. In fact, learning impairment, ${ }^{29-31}$ changes in social behaviors, ${ }^{32}$ and enhancement of anxiety-like behaviors were observed in rat offsprings prenatally exposed to morphine..$^{33}$ Accordingly, the present study compared the effects of prenatal exposure to buprenorphine, methadone, or morphine on cognitive function, social interaction, and anxiety-like behaviors in young adult rats to evaluate whether the buprenorphine or methadone maintenance therapy during pregnancy is beneficial in reducing the risk of mental health problems in the offsprings.

\section{Materials and methods}

\section{Animals}

Pregnant Sprague-Dawley rats (BioLASCO Taiwan Co, Ltd, Taipei, Taiwan) and their offsprings were used in the experiments. The pregnant female rats (at embryonic day 2 [E2]), 10-12 weeks old and weighing 200-250 g, were shipped from an animal breeding company. After arrival, the dams were acclimatized to a room with controlled temperature $\left(25^{\circ} \mathrm{C}\right)$, humidity ( $50 \% \pm 10 \%)$, and a 12-hours day-night cycle (light on 7 am-7 pm) for 24 hours before experimentation. Rat dams during gestation and nursing were kept individually in separate cages, and their offsprings were housed two to three per cage after weaning. All animals were provided with food (Prolab 2500 Rodent 5P14, LabDiet; PMI Nutrition International, St Louis, MO, USA) and water ad libitum. The ethical guidelines provided by Laboratory Animal Center of the National Health Research Institutes were followed throughout the study.

\section{Drugs}

Morphine (NBCD, Taipei, Taiwan), methadone (USP, Rockville, MD, USA), and buprenorphine (Sigma-Aldrich, St. Louis, MO, USA) were dissolved in distilled water and were administrated subcutaneously (sc) in a volume of $1.0 \mathrm{~mL} / \mathrm{kg}$ of body weight. Heroin is a major drug of abuse by addicts and is rapidly converted to morphine after crossing the blood-brain barrier into the central nervous system. Accordingly, we used morphine directly as a test agent in this study.

\section{Animal treatment}

The dose of opioids used in pregnant rats was selected based on the studies reported previously. ${ }^{19-21,34}$ The dosage was selected to produce overt toxicity, but not overdose deaths. Briefly, pregnant Sprague-Dawley rats (at E2) were randomly assigned to different groups and were sc injected with opioids or vehicle during E3-E20. Four experimental groups were established in this study, Group 1 (vehicle control) rats received distilled water $1 \mathrm{~mL} / \mathrm{kg}$, sc, twice a day. Group 2 (buprenorphine) rats received buprenorphine, $3 \mathrm{mg} / \mathrm{kg}$, sc, 
once a day. Group 3 (methadone) rats received methadone, $5 \mathrm{mg} / \mathrm{kg}$ on E3 and $7 \mathrm{mg} / \mathrm{kg}$, sc, twice a day (E4-E20). Group 4 (morphine) rats received morphine, $2 \mathrm{mg} / \mathrm{kg}$ (initial dose) to $4 \mathrm{mg} / \mathrm{kg}$ (final dose), sc, twice a day (increment of $1 \mathrm{mg} / \mathrm{kg}$ per week). All rats received drug injections between $8.30 \mathrm{am}$ and 9 am and between 4.30 pm and 5 pm except Group 2, in which buprenorphine was administered only once in the morning. The offsprings were weaned at postnatal (PN) day 28 and were maintained until use. The offsprings from the same dam were randomly assigned to different experiments to avoid litter effect.

\section{Experiment procedures}

The detailed scheduling of behavioral measurement is shown in Figure 1. All the animals were evaluated by a variety of behavioral tests. Novel object recognition test was examined on PN 44 and 45. Locomotor activity was measured on PN 48. Social interaction test was measured on PN 55. The anxiety-like behaviors were measured on PN 62 (light-dark transition test) and PN 67 (elevated plus-maze test).

\section{Novel object recognition test}

The experimental apparatus consisted of a Plexiglas openfield box $(50 \times 50 \times 46 \mathrm{~cm})$. The procedure consisted of three sessions - habituation, training, and retention - and animals were videotaped during both the training and retention sessions. During the habituation session, the animals were individually habituated to the box, with 10 minutes of exploration in the absence of objects for 2 consecutive days. During the training session, each animal was first placed in the test box for a 5-minute habituation period. Two identical objects were introduced in two corners (approximately $30 \mathrm{~cm}$ apart from each other) and the animal was allowed to explore the box for 5 minutes (day 4). An animal was considered to be exploring the object when its head was facing the object (the distance between the head and the object was approximately $1 \mathrm{~cm}$ or less) or it was touching or sniffing the object. The time spent in exploring each object was recorded by an experimenter blinded to the identity of the treatments, using stopwatches. The animals were immediately taken back to their home cages after the training session. During the retention session 24 hours after the training session, the animals were placed into the same box, but one of the familiar objects used during the training was replaced with a novel object. The objects were different in shape and color but similar in size. The animals were then allowed to explore freely for 5 minutes, and the time spent in exploring each object was recorded as. In the training session, the preference index was calculated as a ratio of the time spent in exploring the object that was replaced by the novel object in the retention session over the total exploring time. A preference index obtained from the retention session, a ratio of the time spent in exploring the novel object over the total time spent in exploring both the objects, was used to measure cognitive function. The box was wiped clean with $70 \%$ ethanol between tests. The animals with total exploring time $<10$ seconds during both sessions were excluded.

\section{Social interaction test}

For the social interaction test, we modified the method described by Bandstra et al. ${ }^{35}$ This protocol was adopted for the evaluation of negative schizophrenic symptom-like

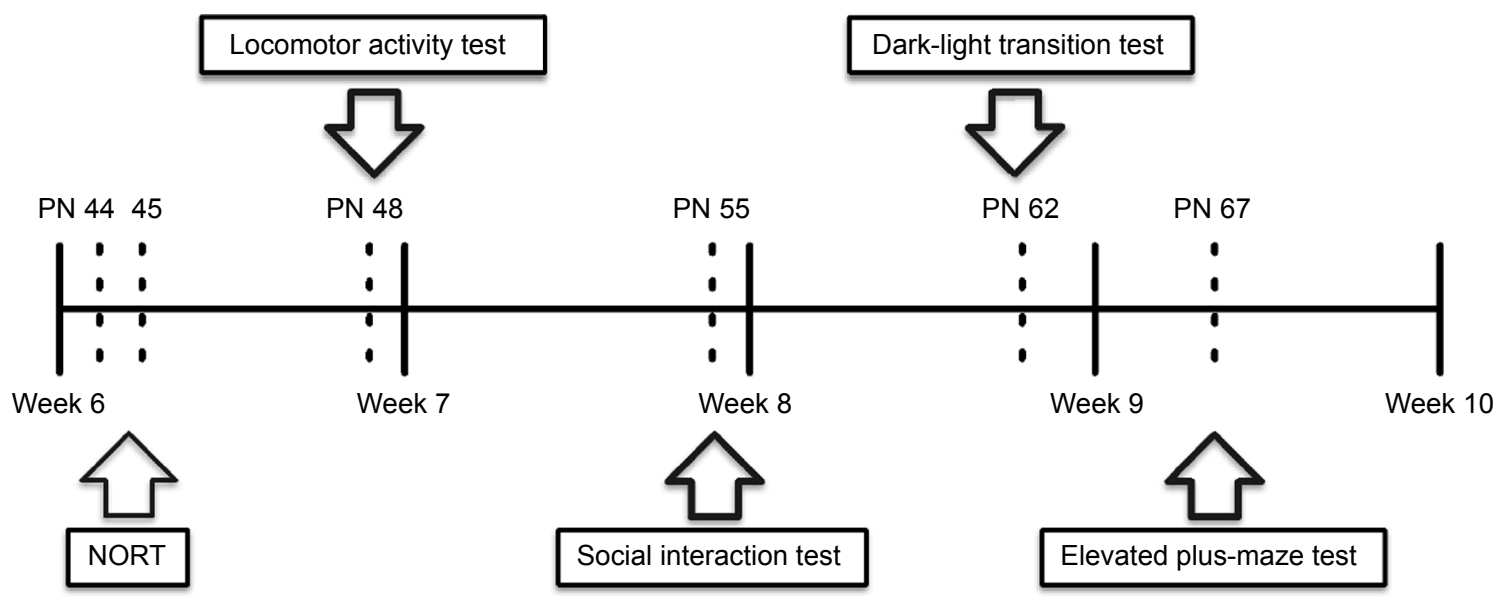

Figure I Experimental schedule.

Notes: Behavioral tests were performed during weeks 6-10. Novel object recognition test was conducted on PN (postnatal day) 44 and 45 . Open-field test was performed on PN 48. Social interaction was measured on PN 55. The anxiety-related behaviors were evaluated on PN 62 (light-dark transition test) and PN 67 (elevated plus-maze test). Abbreviation: NORT, Novel object recognition test. 
behaviors, which was modified from the original social interaction test in that aggressive behaviors (biting and boxing) and passive contact (sitting or lying with bodies in contact) were not included in the social interaction score. The social interaction between pairs of rats was examined in an open-field box $(50 \times 50 \times 46 \mathrm{~cm})$ under normal room lighting.

Every rat was randomly assigned to an unfamiliar partner in the same treatment group. Each pair of rats was placed in the apparatus for 10 minutes and the time that a pair spent in social interaction (sniffing and grooming the partner, following, mounting, and crawling under or over the partner) was recorded by video tracer. The box was wiped clean with $70 \%$ ethanol between tests.

\section{Light-dark transition test}

This test was performed using a chamber $(50 \times 50 \times 50 \mathrm{~cm})$ divided into light and dark compartments. There is an opening allowing animal passage between the two compartments. Before the test, the animals were placed in a behavioral testing room for 30 minutes for adaptation. The light compartment was directly illuminated by a $25-\mathrm{W}$ white light placed $50 \mathrm{~cm}$ above the floor of the compartment. The animals were placed into the light compartment and were made to face the opposite side of the opening. A transition was counted when the animals crossed with their four paws from one side to the other, independent of the direction of the transition. The time spent in the two compartments was measured for 5 minutes. The chamber was wiped clean with $70 \%$ ethanol to thoroughly prevent the interference from the smell of feces and urine after each test.

\section{Elevated plus-maze}

An elevated plus-maze measuring $50 \mathrm{~cm}$ long and $10 \mathrm{~cm}$ wide was constructed of Plexiglas and consisted of four arms (two open arms with 2-cm-high walls and two closed arms with 50 -cm-high walls), $50 \mathrm{~cm}$ long and $10 \mathrm{~cm}$ wide. The cross center is a $10 \times 10-\mathrm{cm}$ square area $\left(100 \mathrm{~cm}^{2}\right)$. The central bottom of the maze was elevated $50 \mathrm{~cm}$ above the floor. Each animal was tested for 5 minutes on the maze, and the behavioral data were automatically recorded by videotape. The animals were placed on the central platform of the maze facing the open arm. The following indices were recorded: the total number of entries into open arms and closed arms and the total time spent in each type of arm. The percentage of time spent in the open arms was calculated for each animal and provided as the measures of anxiety. An entry was defined as the entry of all four feet into one arm and an exit was defined as two paws leaving the arm. Between tests, the maze was wiped clean with $70 \%$ ethanol to thoroughly prevent the interference from the smell of feces and urine.

\section{Locomotor activity in the open field}

The animals were moved from the home cage and put into activity cages $(50 \times 50 \times 46 \mathrm{~cm})$ equipped with computercontrolled photobeams to automatically monitor the movement (TruScan; Coulbourn Instruments, PA, USA). Distance (cm) traveled per 10-minute interval was recorded for 60 minutes. The box was wiped clean with $70 \%$ ethanol between tests.

\section{Statistics}

Results were expressed as mean \pm standard error of the mean. Behavioral data were analyzed by a one-way or two-way ANOVA followed by a post hoc Student-Newman-Keuls test. $P$-value $<0.05$ was considered significant.

\section{Results}

\section{Effects of prenatal exposure to} buprenorphine, methadone, or morphine on the dams and offspring

As shown in Table 1, there was no significant difference in the number of offsprings per litter and sex ratio of offsprings among the different treatment groups. Administration of methadone significantly reduced the body weight gain from E3 to E20 in dams. Body weights of the offsprings on the 1st day

Table I Effects of prenatal exposure to opioids on the dams and offspring

\begin{tabular}{|c|c|c|c|c|}
\hline & \multicolumn{4}{|c|}{ Mean \pm standard error of the mean $(n=12)$} \\
\hline & Saline & Buprenorphine & Methadone & Morphine \\
\hline Number of offsprings per litter & $10.9 \pm 0.75$ & $9.08 \pm 0.35$ & $11.0 \pm 0.65$ & $10.25 \pm 0.43$ \\
\hline Sex ratio of offsprings (male/female) & 1.06 & 1.16 & 0.95 & 0.98 \\
\hline Fatality at birth (\%) & $0.57 \pm 0.57$ & $8.74 \pm 3.17 *$ & $2.72 \pm 1.53$ & $0 \pm 0$ \\
\hline Fatality occurred in the offsprings (\%) (PN 2-I0) & $0 \pm 0$ & $12.8 \pm 6.98$ & $2.4 \pm 1.27$ & $0 \pm 0$ \\
\hline Body weight increase in the dams (g) (E3-E20) & $121.60 \pm 7.34$ & I I $6.09 \pm 5.06$ & $101.73 \pm 4.8^{*}$ & $124.08 \pm 3.70$ \\
\hline Body weight of the offsprings on PN I (g) & $6.89 \pm 0.13$ & $6.78 \pm 0.14$ & $6.38 \pm 0.19^{\#}$ & $6.96 \pm 0.14$ \\
\hline Body weight of the offsprings on PN 7 (g) & $14.06 \pm 0.36$ & $13.37 \pm 0.53$ & $12.78 \pm 0.49$ & $|4.2| \pm 0.4 \mid$ \\
\hline
\end{tabular}

Notes: *Significantly different compared to the saline group, $P<0.05$. "Significantly different compared to the morphine group, $P<0.05$.

Abbreviations: $\mathrm{E}$, embryonic day; PN, postnatal day. 
of birth were observed between the methadone- and morphinetreated groups. By PN7, however, there were no significant differences in pup weight among the treatment groups.

The fatality rate at birth was significantly higher in the prenatally buprenorphine-exposed group than in the other treatment groups. The dams exposed to buprenorphine during pregnancy have a higher propensity of failing to lick or sniff their own pups and eat them instead compared with the dams of other treatment groups, reflecting high fatality rate during PN 2-10.

\section{Effects of prenatal exposure to buprenorphine, methadone, or morphine on the novel object recognition test}

As shown in Figure 2A and B, animals in all opioid treatment groups spent similar exploratory time for two identical objects during each training session. Exposure to morphine, methadone, and buprenorphine significantly reduced the recognition index during retention test sessions in both male and female offsprings $\left(F_{3,71}=41.81, P<0.001\right)$, but no effect of sex and no interaction between treatment and sex was noted. The post hoc comparisons of groups also showed that the males but not the females in the buprenorphine-treated group exhibited a higher recognition index than in the morphine- and methadonetreated groups. These results indicate that prenatal exposure to morphine, methadone, and buprenorphine resulted in impairment of the recognition memory functions.

\section{Effects of prenatal exposure to buprenorphine, methadone, or morphine on the social interaction test}

The total contact time between two animals of the same sex and treatment but from different home cages was monitored as shown in Figure 3. Two-way ANOVA revealed the main effect of opioid treatment $\left(F_{3,56}=35.04, P<0.001\right)$ and $\operatorname{sex}\left(F_{1,56}=12.77, P<0.001\right)$ and a significant interaction between opioid treatment and sex $\left(F_{3,56}=3.82, P<0.05\right)$. Prenatal exposure to morphine, methadone, or buprenorphine significantly decreased the contact time as compared with that of the controls in both male and female offsprings. The methadone-treated male offsprings showed more severe social withdrawal as compared with the animals prenatally exposed to morphine or buprenorphine. The sex effect exists. The contact time for the female offsprings was significantly less than that for male offsprings in the buprenorphine- and morphine-treated groups (Figure 3A and B).

\section{Effects of prenatal exposure to buprenorphine, methadone, or morphine on the light-dark transition test}

Two-way ANOVA on the percentage of time that the animal stayed in the light compartment in the light-dark transition test revealed a main effect of treatment $\left(F_{3,72}=62.19\right.$, $P<0.001)$ and sex $\left(F_{1,72}=5.816, P<0.05\right)$, but no significant interaction between treatment and sex. Overall, female rats spent more time in the light compartment as compared with male rats. However, the post hoc comparisons did not show any significant difference between female and male rats in each treatment group. All three prenatal opioid treatment groups showed significantly reduced the time of stay in the light compartment as compared with the controls (Figure 4). Prenatal morphine exposure produced more remarkable reduction in the percentage of time stayed in the light compartment than the methadone and buprenorphine exposure in both male and female offsprings (Figure 4A and B).
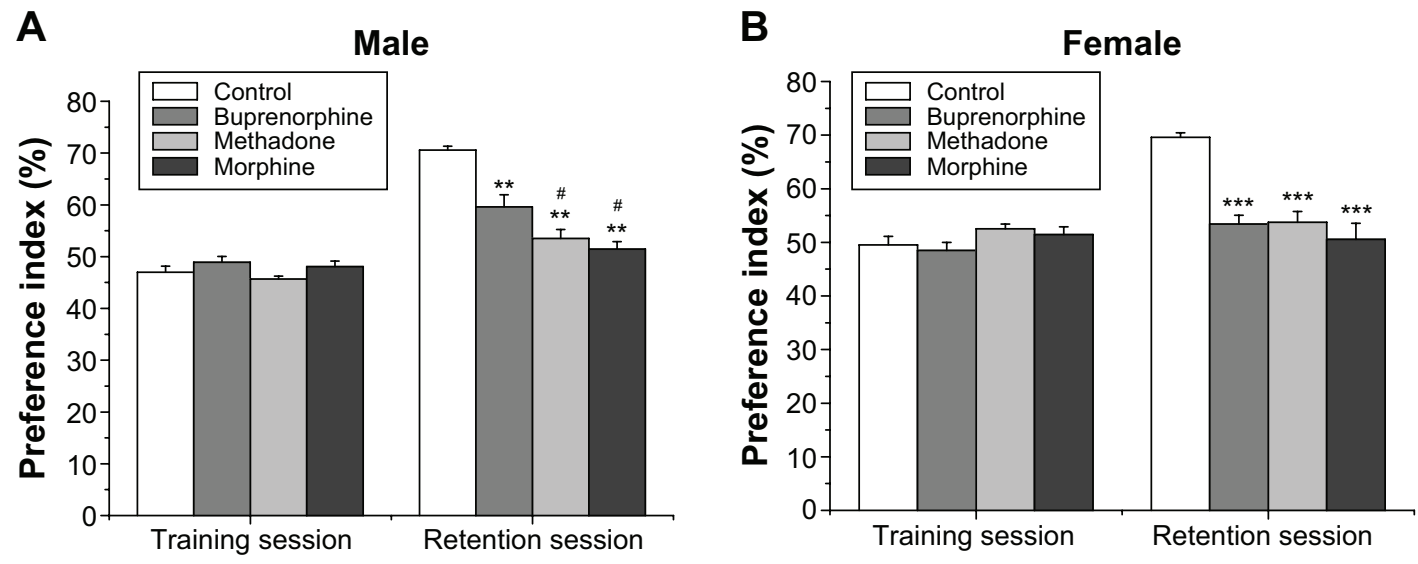

Figure 2 Effects of prenatal exposure to opioids on recognition memory.

Notes: Novel object recognition test was conducted in male (A) and female (B) offsprings. Retention session was carried out 24 hours after the training. The time spent for exploring the objects was measured. The preference index was calculated as a ratio of the time spent in exploring the novel object over the total time spent in exploring both the objects. Values are mean \pm standard error of the mean $(n=9-10) . * * P<0.01$, $* * * P<0.001$ compared with control groups; ${ }^{*} P<0.05$ compared with buprenorphine group. 
A

Male

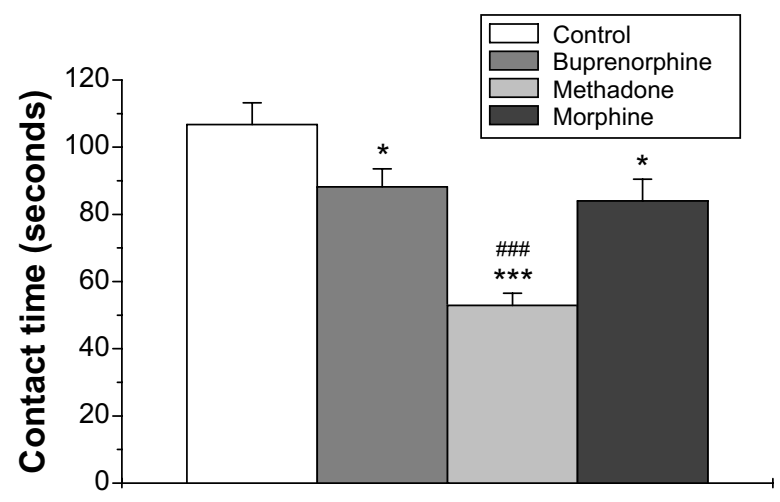

B

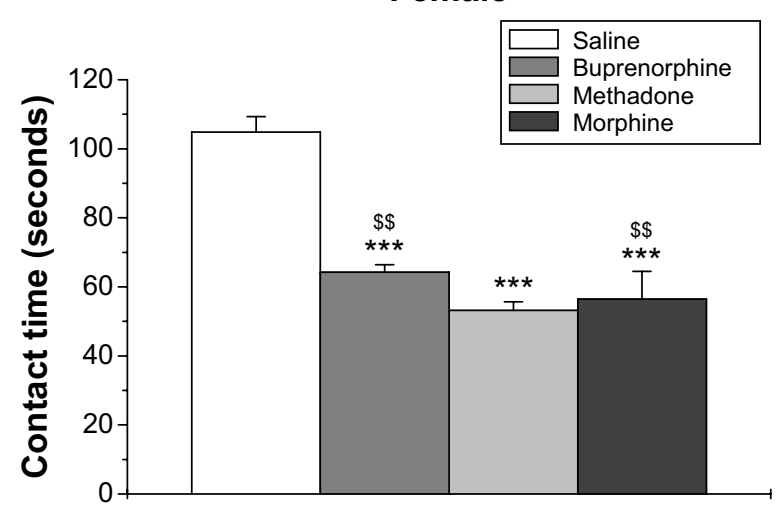

Figure 3 Effects of prenatal exposure to opioids on social behaviors.

Notes: The total social interaction time was recorded in male $(\mathbf{A})$ and female $(\mathbf{B})$ offsprings. All data are expressed as mean $\pm s t a n d a r d$ error of the mean $(n=8)$. $* P<0.05$, $* * * P<0.00$ I compared with control group; ${ }^{\prime \prime \prime} P<0.00$ I compared with morphine or buprenorphine groups; ${ }^{\$ p} P<0.0$ I compared with males with the same treatment.

\section{Effects of prenatal exposure to} buprenorphine, methadone, or morphine
on the elevated plus-maze test

Two-way ANOVA on percentage of time spent in the open arms in the elevated plus-maze revealed a main effect of treatment $\left(F_{3,72}=15.2, P<0.001\right)$ and $\operatorname{sex}\left(F_{1,72}=16.63, P<0.001\right)$, but no interaction between treatment and sex. Female rats spent more time in the open arms as compared with male rats. The post hoc comparisons demonstrated that female offsprings prenatally exposed to saline, methadone, and morphine spent more time in the open arms compared to male offsprings.

As shown in Figure $5 \mathrm{~A}$ and $\mathrm{B}$, prenatal methadone and morphine significantly reduced the percentage of time spent in the open arms in both the sexes. However, the significantly reduced effects of prenatal exposure to buprenorphine on the time spent of open arms was only obtained in the female offsprings (Figure 5B).

\section{Effects of prenatal exposure to buprenorphine, methadone, or morphine on the locomotor activity test}

As shown in Figure 6, the locomotor activity gradually reduced over time. There were no significant differences in the locomotor activities between the different groups prenatally treated with opioids and between the sexes (Figure 6A and B). This result indicates that the basal locomotor activity was not influenced by prenatal exposure to buprenorphine, methadone, or morphine.

\section{Discussion}

The goal of this study was to compare the effects of prenatal exposure to buprenorphine, methadone, and morphine on recognition memory, social interaction, and anxiety-like behaviors during young adulthood. Our results demonstrated that prenatal exposure to these three opioids produced

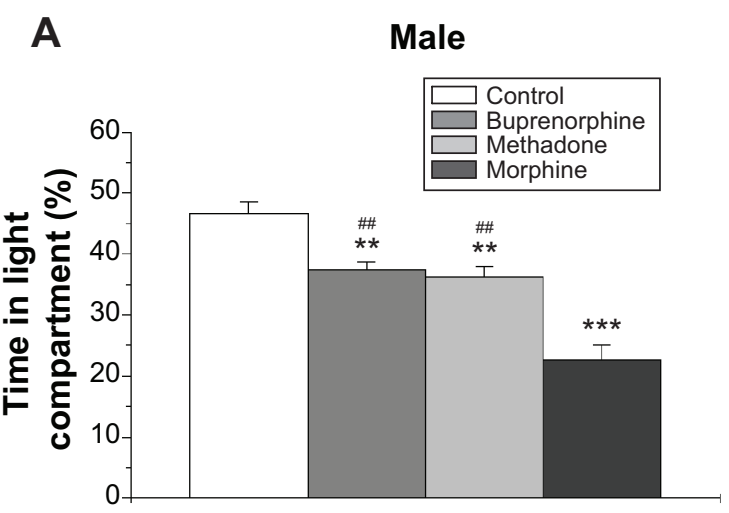

B

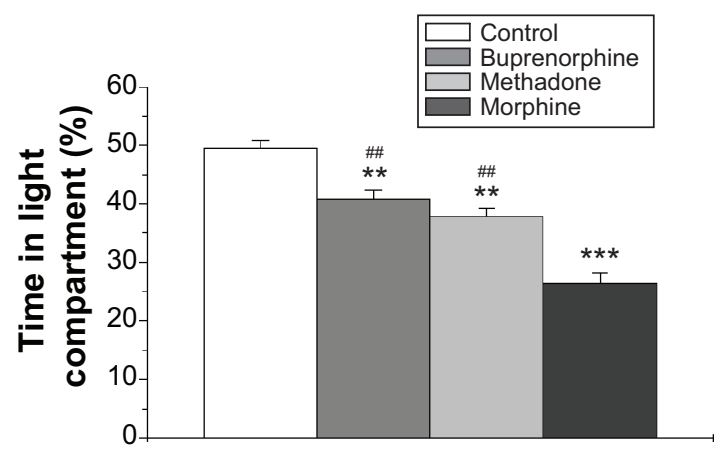

Figure 4 Effects of prenatal exposure to opioids on anxiety-like behavior in the light-dark transition test.

Notes: The percentage of time stayed in the light compartment in the light-dark transition test was recorded in male (A) and female (B) offsprings. All data are expressed as mean \pm standard error of the mean $(n=10)$. ${ }^{*} P<<0.01$, $* * * P<0.001$ compared with control group; ${ }^{*} P<0.01$ compared with morphine group. 

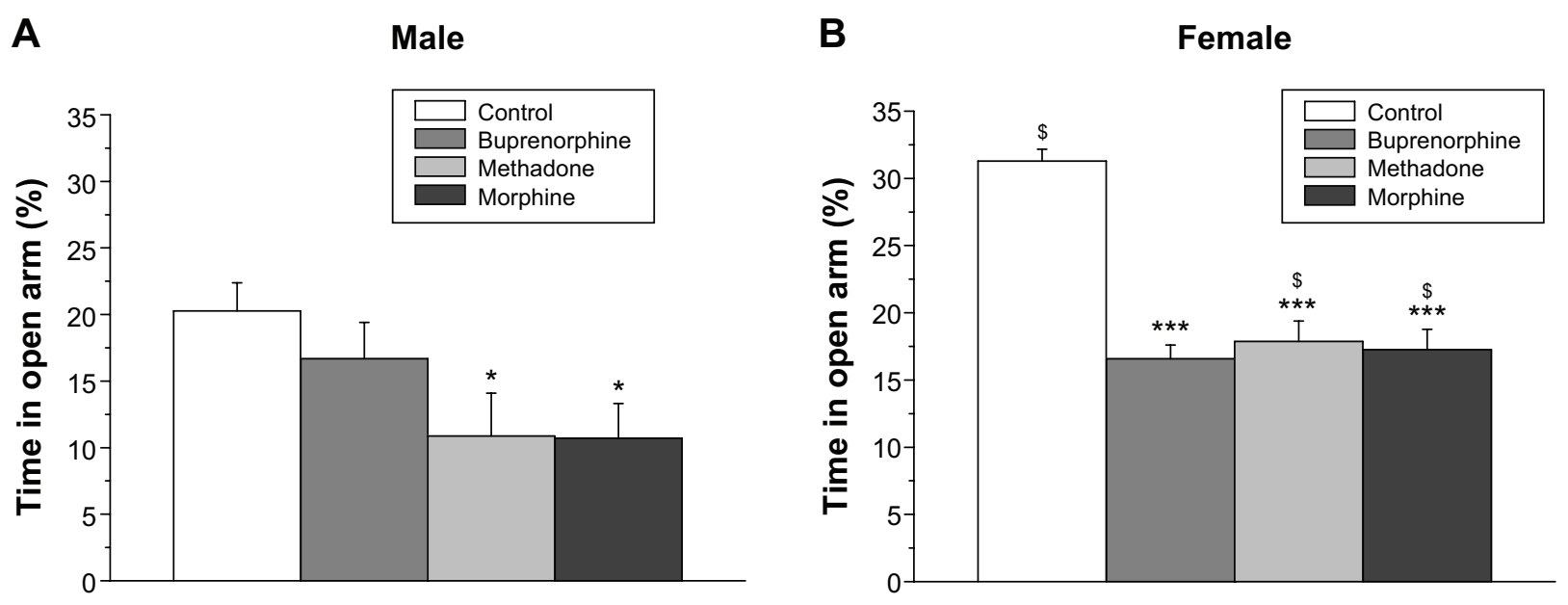

Figure 5 Effects of prenatal exposure to buprenorphine, methadone, and morphine in the elevated plus-maze test.

Notes: The percentage of time spent in the open arms in the elevated plus-maze test was recorded in male (A) and female (B) offsprings. All data are expressed as mean \pm standard error of the mean $(n=10) . * P<0.05, * * * P<0.001$ compared with the control group; ${ }^{\$} P<0.05$ compared with males with the same treatment.

cognitive impairment in the novel object recognition test and reduced social interaction in male and female offsprings. Moreover, rats prenatally exposed to morphine and methadone also showed significant a increase in the index of anxiety-like behaviors in the light-dark transition and elevated plus-maze in both male and female offsprings. However, prenatal buprenorphine exposure spared its effect on the increase of anxiety-like behaviors in the elevated plus-maze in male offspring but not female offsprings. Prenatal exposure to these three opioids did not affect the locomotor activity; thus, the behavioral changes observed in the offsprings were not due to the locomotion deficits.

Several clinical studies demonstrated that children who had intrauterine exposure to heroin or morphine might present long-term neuropsychological consequences caused by dysfunction in intellectual ability and emotional control during their school years. ${ }^{3,436,37}$ Animal studies also suggested that the learning ability and cognitive abilities were reduced in offsprings prenatally exposed to heroin, ${ }^{31,38}$ morphine, ${ }^{31,39}$ L-alpha-acetylmethadol, ${ }^{40}$ or methadone. ${ }^{41-43}$ The current study also revealed that cognitive function of offsprings was impaired after prenatal buprenorphine exposure, suggesting that prenatal exposure to different types of opioids might interfere with learning abilities and memory formation through their common site of action in the opioid system.

Children (boys aged 4-5 years or girls aged 6-11 years) with intrauterine exposure to heroin or heroin/methadone have a higher total behavioral problem score and lower total social competence score than the reference population. ${ }^{44}$ Consistent with the findings obtained from human study, our data showed that the social interaction was significantly reduced in the offsprings that were exposed to methadone, morphine, or buprenorphine. However, previous animal studies
A

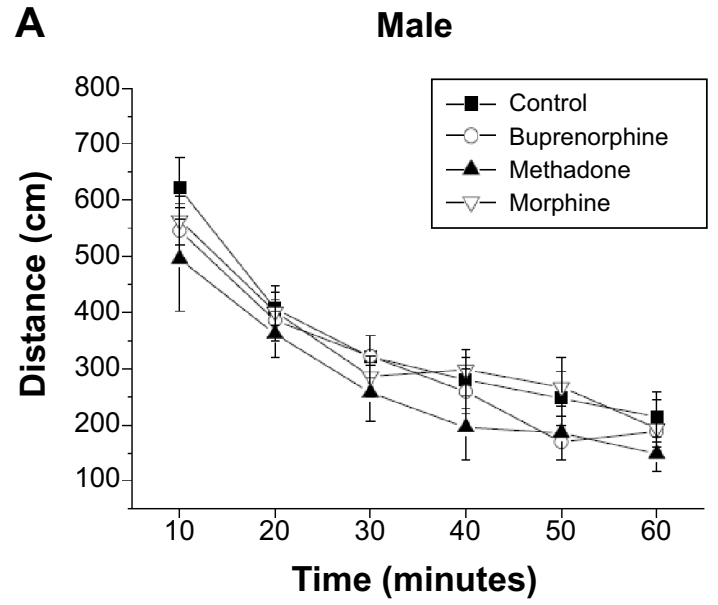

B

Female

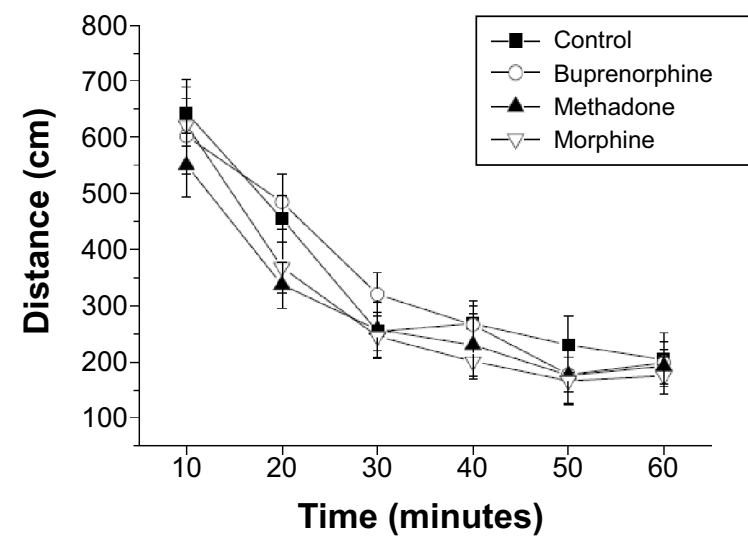

Figure 6 The locomotor activity of offsprings prenatally exposed to buprenorphine, methadone, and morphine in the open-field test.

Notes: The distances traveled in the open field were recorded in male $(\mathbf{A})$ and female $(\mathbf{B})$ offsprings. All data are expressed as mean \pm standard error of the mean ( $\mathrm{n}=10$ ). 
showed higher social behaviors (pinning behavior and social grooming) in offsprings prenatally exposed to morphine than their vehicle control on day 21 and more social approach and less social avoidance during adulthood in the group prenatally exposed to morphine..$^{32,45}$ The controversial results may be due to the different measurement facilities (larger vs small box size) or experimental design (dosage or treatment schedule). In fact, $\mu$ - and $\kappa$-opioid receptors have been implicated in the regulation of social behaviors. ${ }^{26,46}$ Moreover, prenatal exposure to morphine or methadone altered the expression of $\mu$-opioid receptors in the central nervous system. ${ }^{34,47}$ Thus, alterations in $\mu$-opioid receptor function may be associated with the reduced social behaviors.

A recent study showed that prenatally morphine-exposed male offsprings had a tendency of spending less time in the open arms of the elevated plus-maze. ${ }^{48}$ Our results agreed with previous findings that prenatal exposure to morphine had a long-term effect on anxiety-related behaviors, which included the elevated plus-maze and light-dark transition test in both male and female offsprings. The present study further included the measurements of offsprings prenatally exposed to methadone and buprenorphine. Prenatal exposure to methadone or buprenorphine also produced anxiety-like behaviors in the light-dark transition test with less pronounced effect as compared with exposure to morphine. Prenatal methadone exposure significantly increased the anxiety-like behavior in the elevated plus-maze in both sexes, but buprenorphine spared the effects in the male offsprings. Results from previous studies have shown that the sex difference of opioid effects might be due to the influence of the estrogen-induced dimerization of opioid receptors. ${ }^{49}$ However, the reason behind why the offsprings prenatally exposed to buprenorphine or methadone showed less anxietylike behaviors than offsprings prenatally exposed to morphine are unclear and need further investigation. They may be due to the different metabolic pathways (phase 1 vs phase 2) or receptor-binding properties. ${ }^{50-52}$

The present study revealed that methadone and morphine produced comparable effects in most behavioral tests. Buprenorphine exerted less effect on anxiety-like behaviors as compared to morphine and methadone in the elevated maze test. It is notable that buprenorphine pharmacologically differs from methadone; therefore, the effects of buprenorphine on the developing animals may not be totally the same as those of methadone. Buprenorphine is a partial agonist to the $\mu$-opioid receptor, full/partial agonist to nociceptin receptor, and a potent $\kappa$-opioid antagonist. ${ }^{10,11}$
Actually, $\mu$ - and $\kappa$-opioid receptors play distinct roles in regulating anxiety-like behaviors. ${ }^{53-55}$ A recent study also showed that the nociceptin receptor knockout mice exhibited increased anxiety-related behavior in the elevated plus-maze and light-dark box tests. ${ }^{56}$ Although $\mu$-opioid receptors were downregulated after methadone and buprenorphine prenatal treatment, $\kappa 1$-opioid receptors were also upregulated by buprenorphine..$^{47,57,58}$ It remains to be determined whether the discrepancy in opioid receptor adaptation contributes to certain aspects of anxiety-like behaviors.

In summary, our results agreed with most of the previous findings of prenatal morphine exposure on the cognitive function, social interaction, and anxiety-like behaviors. Our study further demonstrated similar behavioral effects were also observed in the offsprings prenatally exposed to methadone, the most widely used agent for opioid maintenance therapy. Like morphine and methadone, buprenorphine exposure during pregnancy also produced cognitive impairment and social withdrawal, whereas it spared the effect on elevated plus-maze in the male offsprings. The current findings suggest that buprenorphine treatment during pregnancy reduced the potential risk for development of certain aspects of psychiatric illness as compared with treatment using morphine or methadone. Lines of evidence have shown enhancement of tolerance to morphine, reduction in nerve growth factor (NGF) contents, deficits of myelination development and oligodendrocyte maturation, and development of sensitization to methamphetamine in the prenatally buprenorphine-exposed offspring. ${ }^{20,21,59-61}$ These findings indicate that it may be necessary to consider whether higher buprenorphine is a really safe therapeutic agent for treating pregnant women. For those reasons, a long-term follow-up would be an important strategy for conducting the early therapy on children prenatally exposed to opioid when they begin to exhibit neurological and learning deficits. This animal study is an important reference for clinical use of buprenorphine in treating pregnant women addicted to heroin.

\section{Acknowledgments}

We thank the support provided by Tzu Chi University (TCIRP100001-01Y2), National Health Research Institutes (NHRI-EX103-10224NC and NHRI-103A1PDCO-1312141), and China Medical University Hospital (DMR-103-023).

\section{Disclosure}

The authors report no conflicts of interest in this work. 


\section{References}

1. Ostrea EM Jr, Ostrea AR, Simpson PM. Mortality within the first 2 years in infants exposed to cocaine, opiate, or cannabinoid during gestation. Pediatrics. 1997;100(1):79-83.

2. Yanai J, Huleihel R, Izrael M, et al. Functional changes after prenatal opiate exposure related to opiate receptors' regulated alterations in cholinergic innervation. Int $J$ Neuropsychopharmacol. 2003;6(3): 253-265.

3. Ornoy A. The impact of intrauterine exposure versus postnatal environment in neurodevelopmental toxicity: long-term neurobehavioral studies in children at risk for developmental disorders. Toxicol Lett. 2003;14(0-141):171-181.

4. Wilson GS, McCreary R, Kean J, Baxter JC. The development of preschool children of heroin-addicted mothers: a controlled study. Pediatrics. 1979;63(1):135-141.

5. Garrido MJ, Troconiz IF. Methadone: a review of its pharmacokinetic/pharmacodynamic properties. $J$ Pharmacol Toxicol Methods. 1999;42(2):61-66.

6. Joseph H, Stancliff S, Langrod J. Methadone maintenance treatment (MMT): a review of historical and clinical issues. Mt Sinai J Med. 2000;67(5-6):347-364.

7. Ludlow JP, Evans SF, Hulse G. Obstetric and perinatal outcomes in pregnancies associated with illicit substance abuse. Aust N Z J Obstet Gynaecol. 2004;44(4):302-306.

8. Pritham UA, Troese M, Stetson A. Methadone and buprenorphine treatment during pregnancy: what are the effects on infants? Nurs Womens Health. 2007;11(6):558-567.

9. Farid WO, Dunlop SA, Tait RJ, Hulse GK. The effects of maternally administered methadone, buprenorphine and naltrexone on offspring: review of human and animal data. Curr Neuropharmacol. 2008;6(2):125-150.

10. Cowan A. Buprenorphine: the basic pharmacology revisited. $J$ Addict Med. 2007;1(2):68-72.

11. Bloms-Funke P, Gillen C, Schuettler AJ, Wnendt S. Agonistic effects of the opioid buprenorphine on the nociceptin/OFQ receptor. Peptides. 2000;21(7):1141-1146.

12. Simmat-Durand L, Lejeune C, Gourarier L. Groupe d' Etudes Grossesse et A. Pregnancy under high-dose buprenorphine. Eur J Obstet Gynecol Reprod Biol. 2009;142(2):119-123.

13. Jones HE, Kaltenbach K, Heil SH, et al. Neonatal abstinence syndrome after methadone or buprenorphine exposure. $N$ Engl $J$ Med. 2010;363(24):2320-2331.

14. Kahila H, Saisto T, Kivitie-Kallio S, Haukkamaa M, Halmesmaki E. A prospective study on buprenorphine use during pregnancy: effects on maternal and neonatal outcome. Acta Obstet Gynecol Scand. 2007;86(2):185-190.

15. Kaltenbach K, Finnegan LP. Developmental outcome of children born to methadone maintained women: a review of longitudinal studies. Neurobehav Toxicol Teratol. 1984;6(4):271-275.

16. Hans SL. Developmental consequences of prenatal exposure to methadone. Ann N Y Acad Sci. 1989;562:195-207.

17. Hutchings DE. Methadone and heroin during pregnancy: a review of behavioral effects in human and animal offspring. Neurobehav Toxicol Teratol. 1982;4(4):429-434.

18. Rosen TS, Johnson HL. Long-term effects of prenatal methadone maintenance. NIDA Res Monogr. 1985;59:73-83.

19. Robinson SE, Wallace MJ. Effect of perinatal buprenorphine exposure on development in the rat. J Pharmacol Exp Ther. 2001; 298(2):797-804.

20. Chiang YC, Hung TW, Lee CW, Yan JY, Ho IK. Enhancement of tolerance development to morphine in rats prenatally exposed to morphine, methadone, and buprenorphine. J Biomed Sci. 2010;17:46.

21. Chiang YC, Hung TW, Ho IK. Development of sensitization to methamphetamine in offspring prenatally exposed to morphine, methadone and buprenorphine. Addict Biol. 2013;19(4):676-686
22. Kamei J, Miyata S, Takahashi M, Saitoh A. Involvement of delta1opioid receptors in the spatial learning impairment in streptozotocininduced diabetic mice. Nihon Shinkei Seishin Yakurigaku Zasshi. 2005;25(5):221-225.

23. Meilandt WJ, Barea-Rodriguez E, Harvey SA, Martinez JL Jr. Role of hippocampal CA3 mu-opioid receptors in spatial learning and memory. J Neurosci. 2004;24(12):2953-2962.

24. Jamot L, Matthes HW, Simonin F, Kieffer BL, Roder JC. Differential involvement of the mu and kappa opioid receptors in spatial learning. Genes Brain Behav. 2003;2(2):80-92.

25. Martel FL, Nevison CM, Simpson MJ, Keverne EB. Effects of opioid receptor blockade on the social behavior of rhesus monkeys living in large family groups. Dev Psychobiol. 1995;28(2):71-84.

26. Vanderschuren LJ, Niesink RJ, Spruijt BM, Van Ree JM. Mu- and kappa-opioid receptor-mediated opioid effects on social play in juvenile rats. Eur J Pharmacol. 1995;276(3):257-266.

27. Perrine SA, Hoshaw BA, Unterwald EM. Delta opioid receptor ligands modulate anxiety-like behaviors in the rat. $\mathrm{Br} J$ Pharmacol. 2006;147(8):864-872.

28. Kudryavtseva NN, Gerrits MA, Avgustinovich DF, Tenditnik MV, Van Ree JM. Modulation of anxiety-related behaviors by mu- and kappaopioid receptor agonists depends on the social status of mice. Peptides. 2004;25(8):1355-1363.

29. Yang SN, Liu CA, Chung MY, et al. Alterations of postsynaptic density proteins in the hippocampus of rat offspring from the morphineaddicted mother: beneficial effect of dextromethorphan. Hippocampus. 2006;16(6):521-530.

30. Nasiraei-Moghadam S, Sherafat MA, Safari MS, Moradi F, Ahmadiani A, Dargahi L. Reversal of prenatal morphine exposureinduced memory deficit in male but not female rats. J Mol Neurosci. 2013;50(1):58-69.

31. Niu L, Cao B, Zhu H, et al. Impaired in vivo synaptic plasticity in dentate gyrus and spatial memory in juvenile rats induced by prenatal morphine exposure. Hippocampus. 2009;19(7): 649-657.

32. Hol T, Niesink M, van Ree JM, Spruijt BM. Prenatal exposure to morphine affects juvenile play behavior and adult social behavior in rats. Pharmacol Biochem Behav. 1996;55(4):615-618.

33. Voronina TA, Chobanov NG, Molodavkin GM, Zhukov VN. Povyshenie trevozhnosti i snizhenie bolevoř chuvstvitel'nosti u potomstva krys posle prenatal'noǐ morfinizatsii [Increase in anxiety and decrease in pain sensitivity in offspring of rats after prenatal morphine exposure]. Biull Eksp Biol Med. 1994;118(8):154-156. Russian.

34. Chiou LC, Yeh GC, Fan SH, How CH, Chuang KC, Tao PL. Prenatal morphine exposure decreases analgesia but not $\mathrm{K}+$ channel activation. Neuroreport. 2003;14(2):239-242.

35. Bandstra ES, Morrow CE, Mansoor E, Accornero VH. Prenatal drug exposure: infant and toddler outcomes. $J$ Addict Dis. 2010;29(2): $245-258$.

36. Topley J, Windsor D, Williams R. Behavioural, developmental and child protection outcomes following exposure to class A drugs in pregnancy. Child Care Health Dev. 2008;34(1):71-76.

37. van Baar AL, Soepatmi S, Gunning WB, Akkerhuis GW. Development after prenatal exposure to cocaine, heroin and methadone. Acta Paediatr. 1994;404:40-46.

38. Golalipour MJ, Ghafari S. Purkinje cells loss in off spring due to maternal morphine sulfate exposure: a morphometric study. Anatomy Cell Biol. 2012;45(2):121-127.

39. Rimanoczy A, Slamberova R, Vathy I. Prenatal morphine exposure alters estrogen regulation of kappa receptors in the cortex and POA of adult female rats but has no effects on these receptors in adult male rats. Brain Res. 2001;894(1):154-156.

40. Schrott LM, Franklin L, Serrano PA. Prenatal opiate exposure impairs radial arm maze performance and reduces levels of BDNF precursor following training. Brain Res. 2008;1198:132-140. 
41. Zagon IS, McLaughlin PJ. Perinatal methadone exposure and brain development: a biochemical study. J Neurochem. 1978;31(1):49-54.

42. Zagon IS, McLaughlin PJ, Thompson CI. Learning ability in adult female rats perinatally exposed to methadone. Pharmacol Biochem Behav. 1979;10(6):889-894.

43. Van Wagoner S, Risser J, Moyer M, Lasky D. Effect of maternally administered methadone on discrimination learning of rat offspring. Percept Mot Skills. 1980;50(3 pt 2):1119-1124.

44. Soepatmi S. Developmental outcomes of children of mothers dependent on heroin or heroin/methadone during pregnancy. Acta Paediatr. 1994;404:36-39.

45. Niesink RJ, van Buren-van Duinkerken L, van Ree JM. Social behavior of juvenile rats after in utero exposure to morphine: dose-time-effect relationship. Neuropharmacology. 1999;38(8):1207-1223.

46. Panksepp J, Bishop P. An autoradiographic map of $(3 \mathrm{H})$ diprenorphine binding in rat brain: effects of social interaction. Brain Res Bull. 1981;7(4):405-410.

47. Darmani NA, Schnoll SH, Pandey U, Martin BR. Chronic prenatal methadone exposure alters central opioid mu-receptor affinity in both fetal and maternal brain. Neurotoxicol Teratol. 1992;14(4):265-271.

48. Klausz B, Pintér O, Sobor M, et al. Changes in adaptability following perinatal morphine exposure in juvenile and adult rats. Eur J Pharmacol. 2011;654(2):166-172.

49. Lee CW, Ho IK. Sex differences in opioid analgesia and addiction: interactions among opioid receptors and estrogen receptors. Mol Pain. 2013;9:45

50. Smith HS. Opioid metabolism. Mayo Clin Proc. 2009;84(7): 613-624.

51. Trescot AM, Datta S, Lee M, Hansen H. Opioid pharmacology. Pain Physician. 2008;11(2 suppl):S133-S153.

52. Lutfy K, Cowan A. Buprenorphine: a unique drug with complex pharmacology. Curr Neuropharmacol. 2004;2(4):395-402.
53. Marín S, Marco E, Biscaia M, et al. Involvement of the kappa-opioid receptor in the anxiogenic-like effect of CP 55,940 in male rats. Pharmacol Biochem Behav. 2003;74(3):649-656.

54. Le Merrer J, Cagniard B, Cazala P. Modulation of anxiety by mu-opioid receptors of the lateral septal region in mice. Pharmacol Biochem Behav. 2006;83(3):465-479.

55. Carr GV, Lucki I. Comparison of the kappa-opioid receptor antagonist DIPPA in tests of anxiety-like behavior between Wistar Kyoto and Sprague Dawley rats. Psychopharmacology. 2010;210(2):295-302.

56. Gavioli EC, Rizzi A, Marzola G, Zucchini S, Regoli D, Calo G. Altered anxiety-related behavior in nociceptin/orphanin FQ receptor gene knockout mice. Peptides. 2007;28(6):1229-1239.

57. Hou Y, Tan Y, Belcheva MM, Clark AL, Zahm DS, Coscia CJ. Differential effects of gestational buprenorphine, naloxone, and methadone on mesolimbic mu opioid and ORL1 receptor G protein coupling. Brain Res Dev Brain Res. 2004;151(1-2):149-157.

58. Belcheva MM, Bohn LM, Ho MT, et al. Brain opioid receptor adaptation and expression after prenatal exposure to buprenorphine. Brain Res Dev Brain Res. 1998;111(1):35-42.

59. Wu VW, Mo Q, Yabe T, Schwartz JP, Robinson SE. Perinatal opioids reduce striatal nerve growth factor content in rat striatum. Eur J Pharmacol. 2001;414(2-3):211-214.

60. Sanchez ES, Bigbee JW, Fobbs W, Robinson SE, Sato-Bigbee C. Opioid addiction and pregnancy: perinatal exposure to buprenorphine affects myelination in the developing brain. Glia. 2008;56(9):1017-1027.

61. Eschenroeder AC, Vestal-Laborde AA, Sanchez ES, Robinson SE, Sato-Bigbee C. Oligodendrocyte responses to buprenorphine uncover novel and opposing roles of mu-opioid- and nociceptin/orphanin FQ receptors in cell development: implications for drug addiction treatment during pregnancy. Glia. 2012;60(1):125-136.
Neuropsychiatric Disease and Treatment

\section{Publish your work in this journal}

Neuropsychiatric Disease and Treatment is an international, peerreviewed journal of clinical therapeutics and pharmacology focusing on concise rapid reporting of clinical or pre-clinical studies on a range of neuropsychiatric and neurological disorders. This journal is indexed on PubMed Central, the 'PsycINFO' database and CAS,

\section{Dovepress}

and is the official journal of The International Neuropsychiatric Association (INA). The manuscript management system is completely online and includes a very quick and fair peer-review system, which is all easy to use. Visit http://www.dovepress.com/testimonials.php to read real quotes from published authors. 